\title{
Ensuring timely thromboprophylaxis on a Medical Assessment Unit
}

\author{
Oluwatosin Akinbobuyi, louise shalders, Tim Nokes
}

To cite: Akinbobuyi 0 , shalders I, Nokes T. Ensuring timely thromboprophylaxis on a Medical Assessment Unit. BMJ Quality Improvement Reports 2016;5:u212414.w4934 doi:10.1136/bmjquality. u212414.w4934

Received 26 May 2016 Revised 20 September 2016

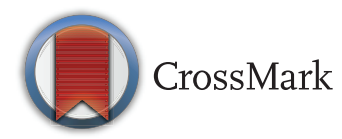

Derriford Hospital, Plymouth Hospitals NHS Trust, United Kingdom

Correspondence to Oluwatosin Akinbobuyi o.akinbobuyi@nhs.net

\section{ABSTRACT}

The Department of Health has defined hospital acquired venous thromboembolism (VTE) as any VTE event occurring within 90 days of hospital admission or surgery. ${ }^{1}$ Hospital acquired thrombosis (HAT) is common during and after hospital admission and is considered a major patient safety issue. Current NICE guideline (CG 92) 2010, recommends that medical patients assessed at risk of VTE should have pharmacological prophylaxis commenced as soon as possible after risk assessment has been completed and continued until the patient is no longer at increased risk of VTE. ${ }^{2}$

This quality improvement project was carried out in the medical assessment unit in Derriford Hospital, Plymouth. We aimed to increase appropriate VTE pharmacological prophylaxis to $100 \%$ prescribed in 6 hours by the end of May 2016 using the Plan-DoStudy-Act (PDSA) methodology. The primary outcome measure was whether or not enoxaparin was given within 6 hours of admission.

Analysis showed that a major contributing factor to the prolonged time interval was lack of awareness of the Trust VTE guidelines which had recently been updated in accordance with NICE recommendations. Baseline measurement demonstrated that $29 \%$ of patients had first dose of enoxaparin within the 6-hour target, with a mean delay of 12 hours 22 minutes. With implementation of an education programme and development of a local VTE prevention care pathway to raise awareness of the new guidelines, a significant improvement was achieved with $71 \%$ of patients meeting the 6 -hour target in PDSA cycle 1 and $83 \%$ of patients in PDSA cycle 2. The average time interval also reduced to 5 hours 52 minutes in PDSA cycle 1 and 5 hours 7 minutes in PDSA cycle 2.

In conclusion, through utilization of simple change methodology, enoxaparin prescribing practice significantly improved. Potentially this may reduce morbidity (and mortality) associated with HAT together with cost implications of its management.

\section{PROBLEM}

In 2005, The House of Commons Health Committee reported that an estimated 25,000 people in the UK die from preventable HAT every year. The inconsistent use of prophylactic measures for VTE in hospital inpatients has been widely reported as a major cause. A UK survey suggested that $71 \%$ of patients assessed to be at medium or high risk of developing VTE did not receive any form of mechanical or pharmacological VTE prophylaxis. $^{23}$

Despite clear NICE guidance on timing of VTE prophylaxis after risk assessment, the majority of patients admitted to the medical assessment unit were routinely prescribed their first dose of pharmacological prophylaxis (enoxaparin) at 18:00, irrespective of when they were admitted. Initial review showed some patients waiting 20 to 24 hours before receiving their first dose of enoxaparin.

This issue was compounded by the old local trust guidelines recommending routine prescription of enoxaparin at 18:00, inadequately completed risk assessments on drug charts and underutilization of the hospital patient information leaflets on VTE which would have created awareness among patients to make informed decisions about their care.

The Trust VTE prevention team utilised root cause analysis in real time as the VTE events occurred. This identified recent significant cases of hospital acquired VTE on MAU and short stay ward. Clearly, these all compromised the recognized standards of care and patient-centred outcomes.

With the high turnover of patients being admitted and transferred from MAU to other medical specialty wards every day and the majority being prescribed enoxaparin outside the 6-hour target, there was clearly a major problem compromising patient safety and high quality care. The aim of the quality improvement project was to increase appropriate VTE pharmacological prophylaxis to $100 \%$ prescribed in 6 hours by the end of May 2016. As NICE guidelines recommends commencement of pharmacological prophylaxis as soon as possible after admission without a particular time target, a 6-hour target was set to ensure that enoxaparin will be given as all drug rounds will be completed during that time. 


\section{BACKGROUND}

Venous Thromboembolism or VTE is a collective name for clots in veins. Usually these develop in the large veins of the legs or pelvis (Deep Vein Thrombosis-DVT) or lungs (Pulmonary Embolism-PE). ${ }^{4}$ Evidence suggests that the risk for VTE extends for at least three months following discharge from hospital (million Women study), subsequently termed Hospital Acquired Thrombosis (HAT) ${ }^{5}$

A Cochrane study in 2009 estimated that two thirds of $\mathrm{PE}$ is hospital-acquired and that $70 \%$ of deaths occur in medical inpatients. The risk of VTE in medical admissions vary from $15 \%$ in general medical patients to $50 \%$ in stroke patients, while clinically recognised $\mathrm{PE}$ occurs in $1 \%{ }^{6}$ It has been recognised in medical patients, that the key risk factors for thrombosis, include personal risk factors such as previous VTE, malignancy, obesity, age over 60, immobility/inactivity. Also acute ill health causing hospitalisation such as sepsis, exacerbation of inflammatory illness and significant medical co-morbidities including heart disease, metabolic, respiratory and endocrine pathologies are all contributory factors. Therefore, the majority of medical patients admitted to hospital have an increased risk for VTE.

Since the House of Commons Health Committee report, a significant number of measures have been introduced to support the prevention of VTE as a priority for the NHS, through the implementation of the National VTE Prevention Programme which provides a comprehensive, integrated and financially incentivised approach to prevent VTE. The Programme includes publication of the key documents: The NICE Clinical Guideline 92, updated January 2010 on venous thromboembolism, The NICE VTE prevention Quality Standard (QS3), The NICE pathway for VTE prevention, the national CQUIN (commissioning for quality and innovation) goal for VTE prevention and a national tool for VTE risk assessment. ${ }^{7}$

The effectiveness of thromboprophylaxis in medical patients has been reviewed by Cochrane [6] and NICE [2]. There are 5 RCT's comparing low molecular weight heparin (LMWH) to placebo in the prevention of $\mathrm{PE}$ in medical patients, and 4 comparing LMWH to unfractionated heparin (UFH). These trials administered prophylactic anticoagulation for up to 10 days. LMWH treatment reduced the incidence of DVT by $60 \%$, and symptomatic PE by $39 \%$ compared to placebo (RR 0.61 , $95 \%$ CI $0.25,1.5){ }^{2}$

Plymouth Hospitals NHS Trust runs Derriford Hospital, the largest hospital in the South West Pennisula providing comprehensive secondary and tertiary health care and serving around 450,000 people in Plymouth, North and East Cornwall and South and West Devon. The Medical Assessment Unit is a 45 bed unit receiving acutely ill medical patients from primary care via GP referral and referral from the Emergency Department. Like most NHS hospitals, Derriford hospital has produced an individualised risk assessment tool for venous thromboembolism. This is based on the national VTE risk assessment tool and is situated in patients' drug chart, which is utilised for all patients admitted to hospital. The local trust guideline on VTE was updated in January 2016, with recommendations according to NICE guidelines. This quality improvement project aimed to increase individual awareness of MAU doctors and nurses to the newly updated trust VTE guideline, through education and development of a trust VTE prevention care pathway to improve the time interval from patient admission to their first dose of pharmacological prophylaxis (enoxaparin).

\section{BASELINE MEASUREMENT}

Baseline measurement: (October 2015).

Prescription charts of in-patients admitted to MAU in October 2015 were reviewed. Data was collected three times per week over a two-week period. The primary outcome measure was whether or not patients had their first dose of enoxaparin within 6 hours of admission. Time of admission in to MAU was noted on the trust IT central patient administration system which is a shared service across the hospital, information on risk assessment completion and time of first enoxaparin prescription was retrieved from the prescription charts.

AVTE red sticker labelled on patient's drug chart also provided extra information on timing of risk assessment completion as this information could not be assessed due to the drug chart design. 31 patients were reviewed. Only 29\% (9 out of 31) of patients had their first dose of enoxaparin within 6 hours of admission. The average time was 12 hours 22 minutes, with some patients waiting over 20 hours before receiving their first dose of thromboprophylaxis. $25 \%$ (8 out of 31 ) of patients had no risk assessment completed and yet $16 \%{ }^{5}$ of them were given enoxaparin (see figure 1).

\section{DESIGN}

The baseline data collection showed that majority of enoxaparin prescriptions were outside the 6-hour target
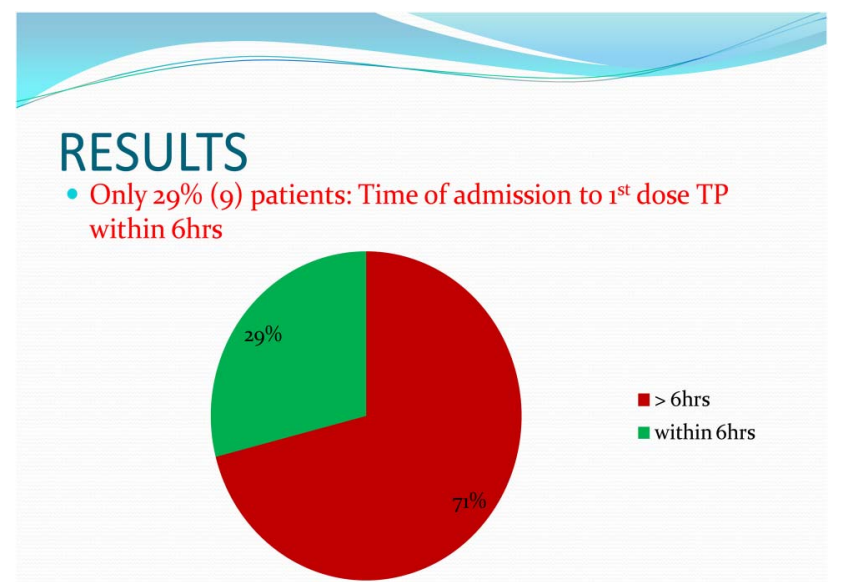

Figure 1 Baseline measurement 
with most of the prescriptions occurring routinely at 18:00 irrespective of patient's time of admission. Working with the Trust VTE lead and Trust VTE clinical nurse specialist, we evaluated the underlying cause of the problem. In order to improve the time interval from admission to first dose of thromboprophylaxis, we decided to survey MAU staff to assess their knowledge of HAT and awareness of the newly updated VTE guidelines using questionnaires. The questionnaires focused on their understanding of HAT, its complications, when it was most likely to occur, when they thought risk assessment should be completed, time enoxaparin should be prescribed and if they were aware of any local guidelines regarding VTE prevention. Results from the questionnaire responses showed that majority of staff had a fair knowledge of HAT, its complications and when risk assessment should be completed. However, the majority of respondents thought enoxaparin should be routinely prescribed at 18:00. Though the majority were aware of a local guideline regarding VTE prevention, no one knew that the trust VTE guidelines had recently been updated and published on the hospital intranet in accordance with NICE guidelines.

By analysing the results of the baseline measurement and questionnaire responses, it was evident that the majority of MAU staff were still following the old VTE guidelines which recommended prescribing enoxaparin at 18:00. The first problem to address was lack of awareness of the newly updated VTE guidelines. From the project team's discussions and review of the baseline data we also identified other factors contributing to the prolonged time interval from admission to first dose of enoxaparin. Inconsistencies with completion of risk assessment on drug charts, ineffective communication during doctors' and nurses' handovers and lack of patient awareness to hospital acquired thrombosis (HAT). Review of two recent cases of HAT in the trust showed that ineffective communication between doctors and nurses on MAU and between the MAU and the medical ward teams was also a problem. Some of the nurses were not aware that a stat dose of enoxaparin had been prescribed on MAU due to lack of communication by the clerking doctor. Though all enoxaparin prescriptions were signed for during drug rounds by the nurses, not all the risk assessments had been completed. Lack of patient awareness was identified as one of the problems as it was discovered from the cases of HAT events that patients were not provided with information leaflets to improve their knowledge of hospital acquired blood clots to allow them to make informed decisions about their care.

We decided to test ways to raise awareness of the newly updated VTE guidelines through introduction of an educational program for all MAU staff using the following strategies: ${ }^{1}$ A departmental presentation to educate staff on HAT, its complications and timing of enoxaparin prescription in accordance with the newly updated VTE guidelines. As one of the NICE quality standard 7 statement recommends, offering patients and carers verbal and written information on VTE prevention as part of the admission and discharge process, other interventions to be suggested during the presentation was inclusion of the already available patient information leaflet (PIL) on VTE in the patient admission pack to raise awareness as this was under-utilized at that time. ${ }^{2}$ Produced a poster of the local VTE prevention care pathway which was strategically placed in the unit to serve as a continuous reminder to complete risk assessments and prescribe enoxaparin as soon as possible in accordance with the new guidelines. ${ }^{3}$ Regular attendance by the team at MAU daily whiteboard morning meetings to maintain awareness of the new guidelines and to improve communication among MAU staff. There is a daily departmental whiteboard meeting of MAU staff with ward managers, senior nurses, MAU junior doctors and MAU consultants on take in attendance, it was decided that during this meeting all pending risk assessments will be collated and communicated to the doctors.

\section{STRATEGY}

\section{PDSA CYCLE 1 (MARCH 2016):}

We aimed to raise awareness of the newly updated and published VTE guidelines through education; the hypothesis being that education would improve staff awareness of the revised guidelines and lead to an improvement in the number of appropriate enoxaparin prescriptions within the 6-hour target.

A4 posters were designed of the local VTE prevention care pathway and strategically placed at different places in the unit detailing ${ }^{1}$ prompt completion of risk assessment during admission clerking, ${ }^{2}$ administering the first dose of enoxaparin as a stat dose once risk assessment is completed, ${ }^{3}$ prescribing subsequent doses as close to 24 hours from that particular time or close to next appropriate drug round ${ }^{4}$ Including VTE alerts as part of doctors' and nurses' handover during patient transfer to the appropriate medical ward (see supplementary file).

Results of the baseline measurement and questionnaire responses were presented at the MAU doctor's multidisciplinary meeting with representatives from the Trust VTE prevention team also present to further reinforce appropriate enoxaparin prescribing. During the meeting, awareness was raised to HAT, its complications and cost implications to the trust. Copies of the VTE prevention care pathway were printed and distributed among staff. Nurses were also encouraged to check VTE risk assessments during their drug rounds and to update other members of the healthcare team during white board meetings of pending risk assessments, enoxaparin yet to be prescribed and to include the patient information leaflet in the patient admission packs. The project team arranged regular visits to MAU attending their 11am departmental whiteboard meetings to reinforce effective communication among MAU staff by 
reminding them of the recommendations of the new guidelines.

Data was collected to measure the impact of this first PDSA cycle. As with the baseline, data was collected three times per week over a two- week period. Of the 34 patients reviewed, all had risk assessments completed and the percentage of patients receiving enoxaparin within 6 hours of admission increased from 29\% to $71 \%$. The mean time reduced from 12 hours 22 minutes to 5 hours 52 minutes. Learning from our first PDSA cycle showed that quality of communication regarding guidelines and staff awareness (or lack of it) has a significant impact on appropriate and timely prescribing.

PDSA CYCLE 2 (May 2016):

The aim of the second cycle was to test whether compliance with timeliness of prescribing would be improved further if staff received feedback. We believed that providing feedback to MAU staff with the positive results of PDSA cycle 1 would increase motivation, drive further improvement and sustain interest and awareness with the new local VTE guidelines. A circular email was sent to MAU staff including all MAU consultants, junior doctors, nurses and ward managers informing them of the encouraging results from PDSA cycle 1. The email also included a copy of the newly updated VTE guidelines and the VTE prevention care pathway. This information was further reinforced by another circular email from the consultant haematologist and the Trust VTE lead to all the MAU staff affirming that the trend of prescribing LMWH at 18:00 should be discontinued and the new local guidelines be strictly adhered to. The MAU consultants circulated further information through email that the new guidelines should be strictly adhered to and anyone still following the old guidelines was doing so at his/her own risk. Ongoing education and feedback continued by regularly attending daily MAU whiteboard meetings.

Data was collected to measure the impact of this PDSA cycle. As before the data was collected three times per week over a two-week period. 23 inpatients on MAU were reviewed. As with PDSA 1, all patients had a VTE risk assessment completed and the percentage of patients receiving enoxaparin within 6 hours increased from $71 \%$ to $83 \%$, with a mean time of 5 hours 7 minutes. The awareness measures appeared to have had a relatively positive impact on VTE risk assessment completion and enoxaparin administration.

\section{RESULTS}

Time Interval from admission to first dose of enoxaparin was measured in October 2015. Of the 31 patients reviewed, only 9 (29\%) achieved the 6-hour target with average time noted to be 12 hours 22minutes with some patients waiting over 20 hours before having their first doses of enoxaparin. 8 out of $31(25 \%)$ had no risk assessments completed and yet $16 \%{ }^{5}$ had enoxaparin prescribed.
Upon implementation of an educational program to raise awareness to the newly updated VTE guidelines in PDSA cycle 1 , there was a significant improvement in the number of patients achieving the 6-hour target from admission to time of first dose of enoxaparin. 34 inpatients on MAU were reviewed in March 2016 over a two-week period. Results showed that $100 \%$ (34) risk assessments were completed, Interval time from admission to first dose enoxaparin meeting the 6-hour target improved from $29 \%$ to $71 \%$ (24 patients) and overall average time improved from 12 hours 22 minutes to 5 hours 52 minutes.

As ongoing education of MAU staff and development of strategically placed posters on VTE prevention care pathway appeared to have had a positive impact on enoxaparin prescribing practice and risk assessments completed, we consolidated on these measures in PDSA cycle 2. We provided feedback to MAU staff by sending emails informing them of the positive results in PDSA cycle 1 to further motivate them to improve enoxaparin prescribing practice and sustain the primary outcome. Data was collected again in May 2016 by reviewing drug prescription charts of 23 inpatients measuring three times per week over a two-week period. Results showed a further improvement from $71 \%$ to $83 \%$ (19) of patients meeting the 6 -hour target and a further improvement in overall average time from 5 hours 52 minutes to 5 hours 7 minutes. It was also noted that all risk assessments remained completed at $100 \%$ (see figure 2).

The implementation of an educational program to raise awareness to the newly updated guidelines saw an improvement in the number of patients achieving the 6-hour target for the interval time from admission to first dose of enoxaparin. The sustainability of these interventions inherently hinges on instilling a personal value in the healthcare team to see VTE prevention as a patient safety issue. Although the initial goal of this quality improvement project was to achieve $100 \%$ appropriate VTE pharmacological prophylaxis by May 2016, $83 \%$ of enoxaparin prescriptions within the 6-hour target has been achieved. There will be need to carry out further PDSA cycles to monitor sustainability of these interventions.

\section{LESSONS AND LIMITATIONS}

A number of lessons have been learnt from carrying out this project. Though the interventions were simple, it was evident that breaking down barriers was critical to success and the implemented measures needed to be thought through carefully to achieve positive results.

For many years it had been Trust practice to prescribe LMWH at 18:00, a major challenge of the project was changing this routine practice. As Health care professionals and practice are generally protocol driven, it was evident from the questionnaire responses that the majority of MAU doctors followed the old trust VTE 
guidelines, which recommended routine prescribing of enoxaparin at 18:00. Hence direct encouragement of MAU staff to change this practice was met by indifference and a lack of motivation, whilst the new guideline was waiting to be updated and published. Upon publication of the new guidelines, implementation of the strategic measures in both PDSA cycles to improve awareness to the newly updated trust VTE guidelines yielded positive results.

Senior clinicians are directly responsible for the clinical safety of their patients and are directly involved with leading and supervision of junior doctors. Involving the MAU consultants in this quality improvement project through regular updates of the results following each PDSA cycle helped ensure that all clerking junior doctors completed risk assessments and enoxaparin prescriptions in accordance with NICE and new Trust VTE guidelines during their post take ward rounds (PTWR). Involvement of the nurses and ward managers also helped to facilitate this change as pending VTE alerts were flagged up to doctors during departmental white board meetings and handovers.

There were a number of limitations to this project. The main challenge we faced was that some doctors expressed concern that prescribing a stat dose during admission clerking may mean that some patients initially risk assessed, receive pharmacological prophylaxis without needing it if they were later subsequently discharged after consultant PTWR. However, it was deemed this was only likely to occur in very few patients with overall benefit of receiving pharmacological prophylaxis outweighing its risk in such situations. Secondly only two
Figure 2 VTE Prevention care pathway

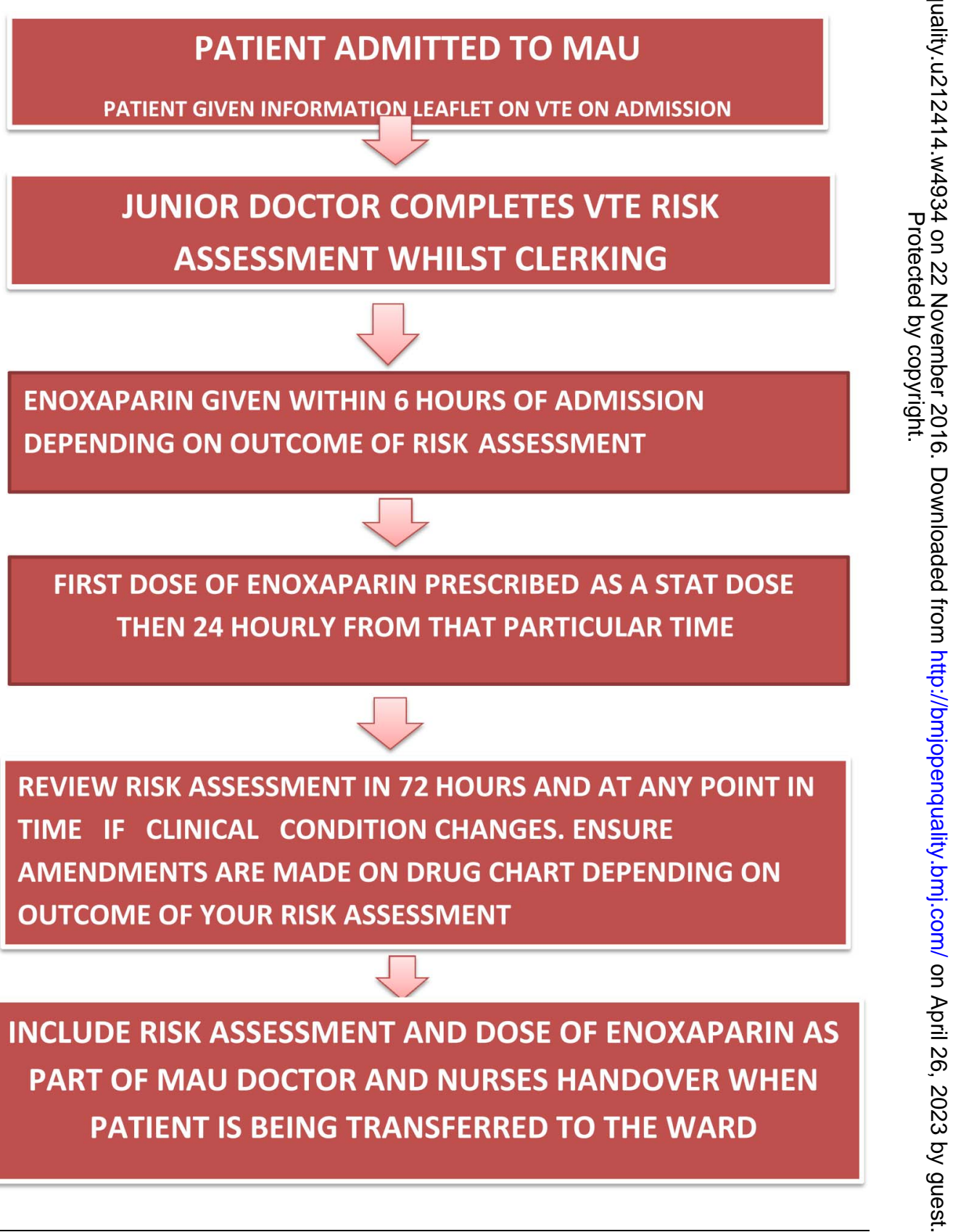


PDSA cycles were carried out, with a smaller sample size in PDSA cycle 2 (this was due to a reduced number of patients meeting the inclusion criteria. Patients already on long term anticoagulation were excluded. A higher percentage of these patients were noted to be admitted in PDSA cycle 2 which meant they had to be excluded). To evaluate effectiveness of the strategic measures and its sustainability, it would be prudent to continue measuring compliance and carry out further PDSA cycles to achieve the target of $100 \%$.

One of the NICE quality standard 7 statement recommends that patients/carers are offered verbal and written information on VTE prevention as part of the admission and discharge process. ${ }^{8}$ We tried to promote this by educating MAU nurses that the already available and under-utilized PIL should be included in the admission pack and given to patients, the implementation and effectiveness of this outcome was not explicitly measured. The literature review also demonstrated that administering prophylactic LMWH led to reduced incidence of DVT and PE, these outcomes were not directly measured during data collection. Though the hospital VTE prevention team investigates all VTE events arising in Trust Inpatients including this as a specific outcome measure in future stages of the project would identify if improvements to the process have an impact on outcome - a reduction in incidence of hospital acquired thrombosis.

Lastly, this quality improvement project only targeted junior doctors currently on MAU placement. Junior doctors regularly rotate through different specialties and with the turnover of a new set of junior doctors every four months, there is a risk to the sustainability of this process. To mitigate against this and to aid sustainability, the results and outcome of the quality improvement project have been presented to the Trust's thrombosis committee. This team is responsible for ensuring that VTE prevention standard operating procedure are up to date, receiving monitoring reports from the hospital VTE prevention team on these standards and overseeing the education of patients and staff on matters concerning VTE prevention. The outcome of their discussion was to continue monitoring compliance to the newly updated Trust VTE guidelines and also continue education by arranging regular visits to their morning departmental meetings, speaking to MAU consultants, junior doctors and ward managers. This is now regularly carried out by the VTE clinical nurse specialist who also reports the results of the monitoring undertaken and recommendations given to the thrombosis committee at every meeting. Feedback from his visits to MAU and audits of drug prescription charts shows that majority of enoxaparin prescriptions are meeting the 6-hour target.

To make further improvements to this process, other ideas have been identified for future tests using the PDSA methodology including measuring effectiveness of including a VTE/risk assessment column in the post take ward round checklist and on the MAU patient whiteboard or putting a VTE assessment alert on the electronic inpatient management system.

The biggest lesson is that it is imperative to have good communication/education regarding changes to key policies and impact of feedback on changing staff behaviour/habits; the need for prompts/aide memoires to support staff to do the right thing.

There are limitations to the generalisability of this project in other specialty admissions units such as surgical assessment unit (SAU) and clinical decision unit (CDU) due to their clinical setting. With SAU admitting surgical patients in the different surgical sub-specialties and CDU being a short-stay multi-specialty ward, applying the 6-hour target for first dose of pharmacological prophylaxis may be impractical as each of these departments have their own adapted risk assessment tools in place for their patients. For example, there is a surgical pathway document for elective surgical patients usually completed in pre-operative assessment clinics and a risk assessment pathway for obstetrics and gynaecological patients.

\section{CONCLUSION}

This quality improvement project aimed to improve time interval from patient admission to their first dose of thromboprophylaxis aiming for 6 hours of admission. One major contributing factor was lack of awareness of the newly updated Trust VTE guidelines. The introduction of interventions to improve awareness of the newly updated guidelines have changed enoxaparin prescribing practice and had a positive impact among health care professionals on the medical assessment unit. However, continuing education of staff is essential to embed a sustainable change in practice that will benefit patients over the long term. This will in turn reduce the risk of hospital acquired thrombosis, its long term complications and cost implications to the hospital and NHS as a whole. Implementation of the interventions in raising awareness can be utilised when guidelines are reviewed or newly introduced.

Acknowledgements Dr Tim Nokes, Consultant Haematologist and Trust VTE Lead. Mr Huw Rowswell, VTE Clinical Nurse Specialist. Mrs Frances Hannon, Service Improvement Lead.

\section{Declaration of interests None Declared.}

Ethical approval According to the policy activities that constitute research at Derriford Hospital, this work met criteria for operational improvement activities exempt from ethics review.It was deemed a quality improvement project and not a study on human subjects, therefore local policy excludes this from requiring ethical approval.

Open Access This is an open-access article distributed under the terms of the Creative Commons Attribution Non-commercial License, which permits use, distribution, and reproduction in any medium, provided the original work is properly cited, the use is non commercial and is otherwise in compliance with the license. See:

- $\mathrm{http}: / /$ creativecommons.org/licenses/by-nc/2.0/

- http://creativecommons.org/licenses/by-nc/2.0/legalcode 


\section{REFERENCES}

1. Arya R, Baglin T, Kakkar A, Fitzmaurice D. Implementation of Thromboprophylaxis in Hospitals. e-Learning for Healthcare. Department of Health, 2010. (accessed 13 December 2015) http:// reception.e-Ifh.org.uk/vte/content/VTE_03_01/d/ELFH_Session/457/ tab_5392.html

2. Reducing the risk of venous thromboembolism (deep vein thrombosis and pulmonary embolism) in patients admitted to hospital. NICE, January 2010.https://www.nice.org.uk/Guidance/cg92 (accessed 15 Mar 2016)

3. The prevention of venous thromboembolism in hospitalised patients. House of Commons Health Committee, 2005. http://webarchive. nationalarchives.gov.uk/+/www.dh.gov.uk/en/Publichealth/ Healthprotection/Bloodsafety/VenousThromboembolismVTE/DH_ 4123668 (accessed 20 Mar 2016)

4. Venous Thromboembolism (VTE) Prevention Standard Operating Procedure; Plymouth Hospitals NHS Trust, Jan 2016.TRW.CLI. SOP.632.2 (accessed 25 Jan 2016)
5. Sweetland S, Green J, Liu B et al.; Million Women Study collaborators. Duration and magnitude of the postoperative risk of venous thromboembolism in middle aged women: prospective cohort study. BMJ 2009 Dec 3;339:b4583. doi: 10.1136/bmj. b4583

6. Alikhan R, Cohen AT. Heparin for the prevention of venous thromboembolism in general medical patients (excluding stroke and myocardial infarction). The Cochrane Database of Systematic Reviews 2009 Jul 8;(3):CD003747. doi: 10.1002/14651858. CD003747.pub2https://www.ncbi.nlm.nih.gov/pubmed/19588346 (accessed 10 Feb 2016)

7. Commissioning Services that deliver High Quality VTE prevention, Guidance for Commissioners, May 2013. https://www.england.nhs. uk/wp-content/uploads/2013/08/vte-prev-guide-may2013-22.7.13.pdf (accessed 10 Mar 2016)

8. Venous thromboembolism prevention quality standard. NICE, June 2010. http://guidance.nice.org.uk/QS3 (accessed 15 Mar 2016) 